\title{
Determinantes da Insegurança Alimentar no Brasil: Análise dos Dados da PNAD de 2004.
}

\author{
Rodolfo Hoffmann ${ }^{1}$
}

\begin{abstract}
São analisados os dados da pesquisa suplementar sobre segurança alimentar, feita pelo IBG E, junto com a Pesquisa Nacional por Amostra de D omicílios - PNAD de 2004, utilizando a Escala Brasileira de Insegurança Alimentar, que classifica os domicílios em quatro categorias: com segurança alimentar ou com insegurança alimentar leve, moderada ou grave. A análise de lógite permite avaliar o efeito de cada fator, controlando o efeito das demais variáveis explanatórias. Contrariamente ao que indica uma análise com apenas uma variável explanatória, verifica-se que residência rural, atividade agrícola e a presença de pessoas com menos de 18 anos no domicílio contribuem para reduzir a probabilidade de insegurança alimentar (IA). 0 determinante mais importante da IA é a baixa renda domiciliar per capita, o que mostra a importância de programas de transferência de renda relativamente bem focalizados, como o bolsa-família. Mesmo depois de controlado o efeito da renda, a escolaridade da pessoa de referência contribui, significativamente, para reduzir a probabilidade de IA. Esta probabilidade aumenta quando a pessoa de referência é preta ou parda, é mulher ou tem ocupação instável e/ ou informal. Também são estimados os efeitos de vários outros determinantes, como região de residência, disponibilidade de água encanada e esgoto, os quais estão associados ao fornecimento de serviços públicos básicos.
\end{abstract}

Palavras-chave: Segurança alimentar, Pobreza, Brasil

\section{Determinants of Food Insecurity in Brazil: Analysis of Data from a 2004 National Survey.}

This paper analyses data from complementary research on food security, carried out by the Brazilian Statistical O ffice - IBGE - jointly with its National Household Sample Survey - PNAD - of 2004. That research was done using the Brazilian Scale of Food Insecurity, which classifies households according to four categories: those with food security, and those with either low, moderate or severe food insecurity. A logit model allows us to assess the impact of each factor while controlling for the impact of the remaining explanatory variables. Contrary to what is suggested by an analysis with a single regressor, it can be observed that to be living in the rural area, to work in the agricultural sector, and the presence in the household of persons with age below 18 years contribute to a lower likelihood of food insecurity (FI). FI's most important determinant is low per capita household income, showing the relevance of governmental transfer programs relatively well focalized, such as the Brazilian family allowances program. Even when controlling for the impact of income, the schooling of the reference person in the household contributes significantly to reduce the probability of FI. Such probability increases if the person of reference is black or mulatto, if that person is a woman, or if he or she has unstable or informal occupation. The paper presents as well estimates of the effects of some other FI determinants, such as the region of residence and the availability of piped water and sanitation, that are associated with the supply of basic public services.

Key words: Food security, Poverty, Brazil.

${ }^{1}$ Instituto de Economia - IE (UNICAMP) 


\section{Introdução}

Pode-se dizer que um domicílio está em situação de segurança alimentar se todas as pessoas deste domicílio têm, permanentemente, acesso a alimentos suficientes para uma vida ativa e saudável.

Na publicação onde divulga os resultados da pesquisa suplementar sobre segurança alimentar, feita juntamente com a PNAD ${ }^{1}$ de 2004, o IBGE ${ }^{[1]}$ cita o texto do Projeto de Lei Orgânica de Segurança Alimentar e Nutricional, em tramitação no Congresso Nacional, que define Segurança Alimentar e Nutricional como:

a realização do direito de todos ao acesso regular e permanente a alimentos de qualidade, em quantidade suficiente, sem comprometer 0 acesso a outras necessidades essenciais, tendo como base práticas alimentares promotoras de saúde, que respeitem a diversidade cultural e que sejam social, econômica e ambientalmente sustentáveis.

É claro que há, necessariamente, certo grau de vagueza no conceito de segurança alimentar, podendo-se qualificar de diversas maneiras os alimentos que devem estar disponíveis para as pessoas. Mas um certo grau de vagueza também é característica de muitos outros conceitos. Considerese, por exemplo, o conceito de pobreza, fortemente associado à insegurança alimentar. Nem por isso, 0 conceito deixa de ser importante e útil.

A situação de extrema insegurança alimentar ocorre quando há grande mortalidade causada pela fome. Em obrajá clássica sobre o tema, Sen ${ }^{[2]}$ analisa vários desses fenômenos, mostrando que eles não são causados pelainexistência de alimentos suficientes, mas pelo fato de as pessoas não terem o direito de acesso (entitlement) aos alimentos.

Nas economias mercantis em geral e, particularmente, na economia brasileira, 0 acesso diário aos alimentos depende, essencialmente, de a pessoa ter poder aquisitivo, isto é, dispor de renda para comprar os alimentos. E uma parcela substancial da população brasileira tem rendimentos muito baixos, determinando a sua insegurança alimentar. Cabe ressaltar que há situações especiais nas quais o acesso a alimentos não depende da renda monetária da pessoa, como é o caso da criança que recebe a merenda escolar ou da produção de alimentos para autoconsumo na agricultura familiar.

Neste trabalho serão analisados os fatores associados à insegurança alimentar, considerando a maneira como ela foi mensurada na pesquisa suplementar da PNAD de 2004. A medida é baseada no número de respostas positivas a um conjunto de 15 perguntas, respondidas por um morador identificado como preparado para respondê-las. Segue a lista das 15 perguntas:

1. Moradores tiveram preocupação de que os alimentos acabassem antes de poderem comprar ou receber mais comida.

2. Alimentos acabaram antes que os moradores tivessem dinheiro para comprar mais comida.

3. Moradores ficaram sem dinheiro para ter uma alimentação saudável e variada.

4. Moradores comeram apenas alguns alimentos que ainda tinham, porque o dinheiro acabou.

5. Algum morador de 18 anos de idade ou mais diminuiu alguma vez a quantidade de alimentos nas refeições ou deixou de fazer alguma refeição, porque não havia dinheiro para comprar comida.

6. Algum morador de 18 anos de idade ou mais alguma vez comeu menos, porque não havia dinheiro para comprar comida.

7. Algum morador de 18 anos de idade ou mais alguma vez sentiu fome, mas não comeu porque não havia dinheiro para comprar comida.

1 Pesquisa Nacional por Amostra de D omicílios. 
8. Algum morador de 18 anos de idade ou mais perdeu peso, porque não comeu quantidade suficiente de comida devido à falta de dinheiro para comprar comida.

9. Algum morador de 18 anos de idade ou mais alguma vez fez apenas uma refeição ou ficou um dia inteiro sem comer, porque não havia dinheiro para comprar comida.

10. Algum morador com menos de 18 anos de idade alguma vez deixou de ter uma alimentação saudável e variada, porque não havia dinheiro para comprar comida.

11. Algum morador com menos de 18 anos de idade alguma vez não comeu quantidade suficiente de comida, porque não havia dinheiro para comprar comida.

12. Algum morador com menos de 18 anos de idade diminuiu a quantidade de alimentos nas refeições, porque não havia dinheiro para comprar comida.

13. Algum morador com menos de 18 anos de idade alguma vez deixou de fazer uma refeição, porque não havia dinheiro para comprar comida.

14. Algum morador com menos de 18 anos de idade alguma vez sentiu fome, mas não comeu, porque não havia dinheiro para comprar comida.

15. Algum morador com menos de 18 anos de idade alguma vez ficou um dia inteiro sem comer, porque não havia dinheiro para comprar comida.

A pontuação atribuída a cada domicílio é o número de respostas afirmativas. Com pontuação igual a zero, o domicílio tem segurança alimentar. No caso dos domicílios cujos moradores têm todos 18 anos de idade ou mais, 1 a 3 pontos corresponde a "insegurança alimentar leve", 4 a 6 pontos corresponde a "insegurança alimentar moderada" e 7 a 9 pontos significa "insegurança alimentar grave". No caso dos domicílios com pelo menos 1 morador de menos de 18 anos de idade, categorias de insegurança alimentar leve, moderada e grave correspondem, respectivamente, aos intervalos de 1 a 5 pontos, 6 a 10 pontos e 11 a 15 pontos.

D essa maneira, obtém-se, para cada domicílio, uma variável "insegurança alimentar" com quatro níveis: ausente, leve, moderada e grave.

\section{Fatores associados à insegurança alimentar nos domicílios}

Para analisar a influência de diversos fatores sobre a insegurança alimentar dos domicílios, foi necessário eliminar, da amostra da PNAD, os domicílios para os quais faltava alguma informação relevante. Assim, foram considerados apenas os domicílios particulares permanentes com informação sobre rendimento domiciliar e a insegurança alimentar. A renda domiciliar per capita é definida como o quociente entre a renda domiciliar e 0 número de pessoas do domicílio, excluindo os pensionistas, empregados domésticos e parentes de empregados domésticos. D este modo, foram excluídas da análise as pessoas que pertenciam a estas três categorias de condição na unidade domiciliar. Foram excluídos os domicílios:

a) sem declaração de escolaridade da pessoa de referência,

b) sem declaração de idade de qualquer pessoa do domicílio,

c) cuja pessoa de referência foi classificada como "indígena" ou "sem declaração de cor",

d) sem declaração sobre existência de água canalizada em pelo menos um cômodo,

e) sem declaração sobre forma de escoadouro do banheiro ou sanitário, e

f) sem declaração sobre forma de iluminação.

Após essas depurações, restou uma amostra de 109.296 domicílios particulares permanentes. Considerando os fatores de expansão fornecidos pelo IBGE, verifica-se que esta amostra representa 
uma população de 50.162.950 domicílios, com 175,56 milhões de pessoas.

Todos os resultados apresentados neste trabalho referem-se a esta amostra, cabendo ressaltar que os cálculos foram feitos ponderando cada domicílio pelo respectivo fator de expansão, sempre utilizando programas em SA S.

A tabela 1 mostra a distribuição dos domicílios por categoria de insegurança alimentar, verificando-se que em 34,9\% há algum grau de insegurança.

Tabela 1. A distribuição dos domicílios conforme categorias de insegurança alimentar. Brasil, 2004.

\begin{tabular}{l|c|c|c|c|c}
\hline \multirow{2}{*}{$\begin{array}{c}\text { Insegurança } \\
\text { alimentar }\end{array}$} & \multicolumn{2}{|c|}{ D omicílios } & \multicolumn{2}{c|}{ Pessoas } & Pessoas \\
\cline { 2 - 5 } & $\begin{array}{c}\text { № } \\
(1.000)\end{array}$ & $\%$ & $\begin{array}{c}\text { № } \\
(1.000)\end{array}$ & $\%$ & $\begin{array}{c}\text { por } \\
\text { domicílio }\end{array}$ \\
\hline Ausente & 32.642 & 65,1 & 105.513 & 60,1 & 3,23 \\
Leve & 8.055 & 16,1 & 31.589 & 18,0 & 3,92 \\
Moderada & 6.196 & 12,3 & 24.898 & 14,2 & 4,02 \\
G rave & 3.270 & 6,5 & 13.557 & 7,7 & 4,15 \\
\hline Total & 50.163 & 100,0 & 175.557 & 100,0 & 3,50 \\
\hline
\end{tabular}

Verifica-se que a proporção de pessoas com segurança alimentar $(60,1 \%)$ é substancialmente mais baixa do que a proporção de domićlios com segurança alimentar, pois nestes é menor o número médio de pessoas por domicílio (3,23 contra mais de 3,9 nos domicílios com algum grau de insegurança alimentar).

Cabe ressaltar que resultados como os apresentados na tabela 1 estão disponíveis na própria publicação do IBGE. ${ }^{[1]}$ Pequenas diferenças numéricas devem-se ao fato de estarmos usando uma amostra depurada de todas as observações onde faltava alguma informação relevante para 0 conjunto da análise que será desenvolvida. Nesse sentido, grande parte das tabelas apresentadas nesta seção poderia ser considerada dispensável. Entretanto, considera-se importante apresentar as associações simples da insegurança alimentar com cada fator, para facilitar a compreensão e mostrar a relevância do modelo de lógite apresentado na próxima seção, por meio do qual é possível considerar, simultaneamente, a influência dos vários determinantes da insegurança alimentar.
A tabela 2 mostra que a insegurança alimentar é maior na área rural do que na área urbana. Isso se deve, essencialmente, à menor renda per capita na área rural. Na próxima seção será verificado que, controlando o efeito da renda e de outros fatores, a insegurança tende a ser menor na área rural do que na área urbana.

Tabela 2. Insegurança alimentar conforme situação do domicílio. Brasil, 2004.

\begin{tabular}{l|c|c|c|c|c}
\hline \multirow{2}{*}{$\begin{array}{c}\text { Situação } \\
\text { do }\end{array}$} & $\begin{array}{c}\text { No de de } \\
\text { domićlios } \\
\text { domicílio }\end{array}$ & \multicolumn{4}{|c}{ Insegurança alimentar (\%) } \\
\cline { 3 - 6 } & Ausente & Leve & Moderada & G rave \\
\hline Urbana & 42.379 & 66,6 & 15,8 & 11,5 & 6,1 \\
Rural & 7.784 & 56,8 & 17,3 & 17,0 & 9,0 \\
\hline Total & 50.163 & 65,1 & 16,1 & 12,3 & 6,5 \\
\hline
\end{tabular}

A tabela 3 mostra como a distribuição dos domicílios pelas categorias de insegurança alimentar é afetada pela presença ou não de pessoas com menos de 18 anos de idade. Os dados indicam que a presença dessas pessoas aumenta a insegurança alimentar. N esse caso, também é importante considerar o efeito da renda per capita, que é menor para domicílios com pessoas com menos de 18 anos. Na próxima seção, será observado que, controlando o efeito da renda domiciliar per capita e de outros fatores, a insegurança alimentar tende a ser menor nos domicílios com pessoas menores de 18 anos.

Tabela 3. Insegurança alimentar conforme 0 domicílio tenha ou não pessoas com menos de 18 anos. Brasil, 2004.

\begin{tabular}{c|c|c|c|c|c}
\hline \multirow{2}{*}{$\begin{array}{c}\text { Presença } \\
\text { de pessoas }\end{array}$} & \multirow{2}{*}{$\begin{array}{c}\text { No de } \\
\text { com idade }\end{array}$} & \multicolumn{4}{|c}{ Insegurança alimentar (\%) } \\
\cline { 3 - 6 } (mil) & 18 & Ausente & Leve & Moderada & G rave \\
\hline Não & 20.124 & 75,7 & 9,7 & 9,1 & 5,5 \\
Sim & 30.039 & 58,0 & 20,3 & 14,5 & 7,2 \\
\hline Total & 50.163 & 65,1 & 16,1 & 12,3 & 6,5 \\
\hline
\end{tabular}

0 número de pessoas por domicílio varia de 1 a 19, com valor médio igual a 3,50 e mediana e moda iguais a 3. A tabela 4 mostra a classificação dos domicílios em 6 estratos de número de pessoas por domicílio, observando-se que a proporção de domicílios com segurança alimentar diminui à medida que aumenta esse número de pessoas. 
Tabela 4. Insegurança alimentar conforme o número de pessoas por domicílio. Brasil, 2004.

\begin{tabular}{c|c|c|c|c|c}
\hline \multirow{2}{*}{$\begin{array}{c}\text { No de } \\
\text { pessoas } \\
\text { por } \\
\text { domicílio }\end{array}$} & $\begin{array}{c}\text { No de } \\
\text { domicílios } \\
\text { (mil) }\end{array}$ & \multicolumn{4}{|c}{ Insegurança alimentar (\%) } \\
\cline { 3 - 6 } & Ausente & Leve & Moderada & G rave \\
\hline 1 & 5.418 & 75,1 & 8,6 & 8,3 & 8,0 \\
2 & 9.742 & 73,6 & 11,1 & 10,0 & 5,3 \\
3 & 11.717 & 68,7 & 16,3 & 10,9 & 4,1 \\
4 & 11.702 & 65,1 & 18,1 & 11,7 & 5,1 \\
5 ou 6 & 8.991 & 53,7 & 21,1 & 16,8 & 8,4 \\
7 ou mais & 2.592 & 34,9 & 22,1 & 23,9 & 19,1 \\
\hline Total & 50.163 & 65,1 & 16,1 & 12,3 & 6,5 \\
\hline
\end{tabular}

A tabela 5 mostra que a insegurança alimentar é maior nos domicílios em que a pessoa de referência é uma mulher do que nos domiclíios em que a pessoa de referência é um homem.

Tabela 5. Insegurança alimentar conforme sexo da pessoa de referência do domicílio. Brasil, 2004.

\begin{tabular}{c|c|c|c|cc}
\hline \multirow{2}{*}{$\begin{array}{c}\text { Pessoa de } \\
\text { referência }\end{array}$} & No de & \multicolumn{5}{|c}{ Insegurança alimentar (\%) } \\
\cline { 3 - 6 } & dmil) & Ausente & Leve & Moderada & G rave \\
\hline Homem & 36.883 & 66,8 & 15,8 & 11,6 & 5,8 \\
Mulher & 13.280 & 60,2 & 16,8 & 14,5 & 8,5 \\
\hline Total & 50.163 & 65,1 & 16,1 & 12,3 & 6,5 \\
\hline
\end{tabular}

Ao analisar como a insegurança alimentar varia com o setor de ocupação da pessoa de referência da família, verificou-se que ela é maior para os ocupados no setor agrícola (agropecuária), como mostra a tabela 6. De acordo com o que será visto na próxima seção, esse é mais um exemplo de um fator cujo efeito muda de sinal quando a análise inclui controles para o efeito da renda domiciliar per capita e outros determinantes da insegurança alimentar.
Tabela 6. Insegurança alimentar conforme o setor de ocupação da pessoa de referência do domicílio ser ou não o agrícola (agropecuária). Brasil, 2004.

\begin{tabular}{c|c|c|c|c|c}
\hline \multirow{2}{*}{$\begin{array}{c}\text { O cupação } \\
\text { agrícola }\end{array}$} & $\begin{array}{c}\text { No de } \\
\text { domicílios } \\
\text { (mil) }\end{array}$ & \multicolumn{4}{|c}{ Insegurança alimentar (\%) } \\
\cline { 3 - 6 } & Ausente & Leve & Moderada & G rave \\
\hline Não1 & 42.274 & 67,1 & 15,7 & 11,3 & 5,9 \\
Sim & 7.889 & 54,1 & 18,0 & 17,8 & 10,1 \\
\hline Total & 50.163 & 65,1 & 16,1 & 12,3 & 6,5 \\
\hline${ }^{1}$ Inclui ocupação na indústria e nos serviços, ramo de atividade \\
não-declarado e inativos.
\end{tabular}

A tabela 7 mostra como a insegurança alimentar varia com o nível da renda domiciliar per capita (RDPC) no domicílio. São considerados 8 estratos de RD PC, delimitados com base no salário mínimo, cujo valor era $\mathrm{R} \$ 260,00$ (em setembro de 2004, que é o mês de referência da PNAD). Verificase que é muito forte a associação entre segurança alimentar e a RDPC. No primeiro estrato, com RDPC igual ou menor do que 1/4 do salário mínimo, a proporção de domicílios com segurança alimentar não chega a $20 \%$ e mais de $27 \%$ tem insegurança alimentar grave. Já no estrato no qual o RD PC ultrapassa 10 salários mínimos, praticamente todos os domicílios têm segurança alimentar.

Tabela 7. Insegurança alimentar em 8 estratos de renda domiciliar per capita. Brasil, 2004.

\begin{tabular}{c|c|c|c|c|c}
\hline \multirow{2}{*}{$\begin{array}{c}\text { Estratos } \\
\text { de RD PC }\end{array}$} & \multirow{2}{*}{$\begin{array}{c}\text { No de } \\
\text { (limites em }\end{array}$} & \multicolumn{4}{|c}{ Insegurança alimentar (\%) } \\
\cline { 3 - 6 } sal. min.) & (mil) & Ausente & Leve & Moderada & G rave \\
\hline$[0 ; 0,25]$ & 4.502 & 19,3 & 20,8 & 32,6 & 27,3 \\
$(0,25 ; 0,5]$ & 8.012 & 36,4 & 26,3 & 25,1 & 12,2 \\
$(0,5 ; 1]$ & 13.532 & 60,4 & 20,6 & 13,6 & 5,4 \\
$(1 ; 2]$ & 12.288 & 78,5 & 13,5 & 5,8 & 2,2 \\
$(2 ; 3]$ & 4.644 & 89,6 & 7,2 & 2,3 & 0,9 \\
$(3 ; 5]$ & 3.696 & 93,9 & 4,6 & 1,2 & 0,3 \\
$(5 ; 10]$ & 2.366 & 97,0 & 2,3 & 0,4 & 0,3 \\
$>10$ & 1.121 & 99,0 & 0,8 & 0,2 & 0,0 \\
\hline Total & 50.163 & 65,1 & 16,1 & 12,3 & 6,5 \\
\hline
\end{tabular}




\section{Modelos de lógite para a insegurança alimentar nos domicílios}

Como os dados que estão sendo analisados não são experimentais, é necessário recorrer a métodos estatísticos relativamente sofisticados, para tentar avaliar a influência de cada fator na insegurança alimentar, se forem mantidos constantes os demais fatores relevantes. É possível fazer isso com um modelo de lógite.

Seja $P_{i}$ a probabilidade de 0 i-ésimo domicílio apresentar insegurança alimentar grave e sejam $x_{h i}$, com $h=1, \ldots, k$, as $k$ variáveis explanatórias que serão consideradas. Então 0 modelo de lógite para insegurança alimentar grave pode ser representado pelo seguinte par de equações:

$$
\begin{aligned}
& P_{i}=\frac{1}{1+\exp \left(-y_{i}\right)} \\
& \text { com } \\
& y_{i}=\alpha+\beta_{1} x_{1 \mathrm{i}}+\beta_{2} x_{2 i}+\ldots+\beta_{k} x_{k i}
\end{aligned}
$$

Alternativamente, o modelo pode ser representado pela equação

$$
\ln \frac{P_{i}}{1-P_{i}}=\alpha+\beta_{1} x_{1 \mathrm{i}}+\beta_{2} x_{2 i}+\ldots+\beta_{k} x_{k i}
$$

Se, por exemplo, o valor de $x_{2}$ aumentar de 1 unidade, mantidas constantes as demais variáveis explanatórias, o valor de $\ln \left[P_{i} /\left(1-P_{i}\right)\right]$ aumenta de $\beta_{2}$, o que corresponde a multiplicar a relação $P_{i} /\left(1-P_{i}\right)$ por $\exp \left(\beta_{2}\right)$, que é a odds ratio associada à variável $x_{2}$.

Na tabela 8 estão os resultados obtidos com um modelo de lógite com apenas sete variáveis explanatórias binárias, usadas para distinguir os 8 estratos de RDPC já descritos na tabela 7. 0 estrato mais pobre (RDPC igual ou menor do que 0,25 salário mínimo) é adotado como base. Para os demais estratos há uma variável binária específica, que assume valor 1 naquele estrato e é igual a zero para os outros estratos.

Tabela 8. Modelo de lógite para a probabilidade de um domicílio ter insegurança alimentar grave. Brasil, 2004.

\begin{tabular}{rc|c|c}
\hline Variável & $\begin{array}{c}\text { Estimativa } \\
\text { do } \\
\text { parâmetro }\end{array}$ & $\begin{array}{c}\text { Estimativa } \\
\text { do desvio } \\
\text { padrão }\end{array}$ & 0 dds ratio \\
\hline Constante & $-0,9784$ & 0,0227 & - \\
$\operatorname{RDPC}^{1}(0,25 ; 0,5]$ & $-0,9960$ & 0,0324 & 0,369 \\
$(0,5 ; 1]$ & $-1,8844$ & 0,0343 & 0,152 \\
$(1 ; 2]$ & $-2,8082$ & 0,0473 & 0,060 \\
$(2 ; 3]$ & $-3,7290$ & 0,1080 & 0,024 \\
$(3 ; 5]$ & $-4,7797$ & 0,2002 & 0,008 \\
$(5 ; 10]$ & $-5,0181$ & 0,2809 & 0,007 \\
$>10$ & $-7,5648$ & 1,4498 & $<0,001$ \\
\hline
\end{tabular}

${ }^{1}$ Renda domiciliar per capita : estratos delimitados em salários mínimos correntes, adotando como base o estrato com RD PC menor ou igual a 0,25 salário mínimo.

Todas as estimativas dos parâmetros, apresentadas na tabela 8, são estatisticamente diferentes de zero. Nos oito casos, a probabilidade caudal associada ao teste da hipótese de nulidade do parâmetro é inferior a 0,0001.

Pelo fato de esse modelo de lógite incluir apenas variáveis explanatórias para distinguir os estratos de RD PC, as estimativas dos parâmetros e as odds ratios podem ser obtidas das informações apresentadas na tabela 7 .

Consideremos, por exemplo, o 3- estrato, com RDPC de mais de 0,5 a 1 salário mínimo. Na tabela 7, verifica-se que, para insegurança alimentar grave, a relação $P_{i} /\left(1-P_{i}\right)$ ou odds nesse estrato é

$$
\frac{0,054}{1-0,054}=0,0571
$$

No estrato mais pobre, tomado como base, essa relação (odds) é 


$$
\frac{0,273}{1-0,273}=0,3755
$$

Verifica-se que

$$
\frac{0,0571}{0,3755}=0,152,
$$

isto é, a relação $P_{i} /\left(1-P_{i}\right)$ no 30 estrato corresponde a apenas 15,2\% (menos de 1/6) do valor dessa relação no $1^{\circ}$ estrato. Esse é 0 efeito de passar do 10 para 0 3o estrato na relação $P_{i} /\left(1-P_{i}\right)$, sendo $P_{i}$ a probabilidade de um domicílio ter insegurança alimentar grave. Note que 0,152 é a odds ratio associada, na tabela 8, ao coeficiente do 30 estrato, pois

$$
\exp (-1,8844)=0,152
$$

Na tabela 9 são apresentados os resultados de um modelo de lógite mais completo, para avaliar a contribuição de vários fatores na determinação da insegurança alimentar grave no país. Na tabela 10 estão os resultados de um modelo com as mesmas variáveis explanatórias, mas procurando explicar a incidência de insegurança alimentar leve, moderada ou grave. ${ }^{2}$

Comparando os coeficientes (ou as odds ratios) referentes aos estratos de renda nas tabelas 8 e 9 , verifica-se que os valores absolutos são menores na tabela 9. Isso é usual e deve-se ao fato de que na tabela 8 as variáveis para estratos de RD PC estão captando os efeitos de outras variáveis, como escolaridade e região de residência, que foram omitidas nessa análise mais simples.

Cabe ressaltar que a renda é uma variável especialmente sujeita a erros de medida. As pessoas esquecem componentes da sua renda e, em particular no caso de rendas relativamente elevadas, tendem a subdeclarar os valores. Além disso, a renda captada na PNAD exclui, explicitamente, o valor da produção para autoconsumo, que pode ser uma parcela substancial da renda de um pequeno agricultor. É provável que, se pudesse ser utilizada uma melhor medida da RD PC, seria observada uma relação ainda mais estreita entre ela e a prevalência de insegurança alimentar.

Foi incluída, como variável explanatória, a escolaridade da pessoa de referência do domicílio. Para a categoria dos "15 anos ou mais", essa variável foi fixada em 17. D esta maneira, o valor médio dessa variável na população analisada é 6,2 e a mediana é 5. De acordo com os resultados apresentados nas tabelas 9 e 10, um ano adicional na escolaridade da pessoa de referência do domicílio reduz a odds [a relação $P_{i} /\left(1-P_{i}\right)$ ] de insegurança alimentar grave em $8,0 \%$ e reduz a odds de insegurança alimentar leve, moderada ou grave em $4,3 \%$.

Já foi constatado, na tabela 5 , que 0 fato de a pessoa de referência do domicílio ser mulher aumenta a prevalência de insegurança alimentar (ver também IBGE $E^{[1]}$, p. 37). 0 sentido desse efeito é confirmado pelos resultados dos modelos de lógite apresentados nas tabelas 9 e 10: o fato de a pessoa de referência do domicílio ser mulher aumenta em cerca de $50 \%$ a odds [a relação $P_{i} /\left(1-P_{i}\right)$ ] de insegurança alimentar. 0 fenômeno não parece estar associado ao nível de renda. Q uando a pessoa de referência é mulher, o valor médio da RD PC (R\$ 501) é até um pouco mais alto do que quando é homem (R\$481). Seria apropriado tentar explicar o fenômeno considerando a natureza algo "subjetiva" da medida de insegurança alimentar utilizada? O efeito se mantém se for usada uma medida mais objetiva? (baseada, por exemplo, em medidas antropométricas). 0 tema demanda mais pesquisas.

Mesmo depois de controlar os efeitos de todas as demais variáveis explanatórias incluídas no modelo, verifica-se que a região de residência afeta substancialmente a probabilidade de insegurança alimentar. Na tabela 9, verifica-se que a odds de insegurança alimentar grave no Estado de São Paulo é igual a 3/4 dessa odds no Nordeste. Q uando se

\footnotetext{
${ }^{2} \mathrm{O}$ coeficiente c, que é uma medida da qualidade do ajustamento do modelo baseada no número da pares concordantes (pares de observações para os quais a ordenação conforme probabilidades estimadas é igual à ordenação conforme valores observados da variável dependente), é igual a 0,844 para a equação da tabela 9 e 0,814 para a equação da tabela 10 .
} 
Tabela 9. Modelo de lógite para a probabilidade de um domicílio ter insegurança alimentar grave. Brasil, 2004.

\begin{tabular}{|c|c|c|c|c|}
\hline Variável & $\begin{array}{l}\text { Estimativa do } \\
\text { parâmetro }\end{array}$ & $\begin{array}{c}\text { Estimativa do desvio } \\
\text { padrão }\end{array}$ & $\begin{array}{l}\text { Prob. } \\
\text { caudal } 1\end{array}$ & 0 dds ratio \\
\hline Constante & $-0,9572$ & 0,1064 & $*$ & - \\
\hline $\mathrm{RD} \mathrm{PC}^{2}(0,25 ; 0,5]$ & $-0,7583$ & 0,0365 & $*$ & 0,468 \\
\hline$(0,5 ; 1]$ & $-1,6067$ & 0,0432 & $*$ & 0,201 \\
\hline$(1 ; 2]$ & $-2,3647$ & 0,0586 & $*$ & 0,094 \\
\hline$(2 ; 3]$ & $-3,1189$ & 0,1156 & $*$ & 0,044 \\
\hline$(3 ; 5]$ & $-4,0322$ & 0,2054 & $*$ & 0,018 \\
\hline$(5 ; 10]$ & $-4,0419$ & 0,2862 & $*$ & 0,018 \\
\hline$>10$ & $-6,4818$ & 1,4499 & $*$ & 0,002 \\
\hline $\mathrm{PR}^{3}$ E scolaridade & $-0,0833$ & 0,0045 & $*$ & 0,920 \\
\hline $\mathrm{PR}^{3}$ Mulher & 0,3953 & 0,0333 & $*$ & 1,485 \\
\hline $\mathrm{PR}^{3}>55$ anos & $-0,2155$ & 0,0409 & $*$ & 0,806 \\
\hline $\mathrm{PR}^{3}$ preto ou pardo & 0,3492 & 0,0308 & $*$ & 1,418 \\
\hline $\mathrm{PR}^{3}$ amarelo & 0,1831 & 0,2821 & $51,6 \%$ & 1,201 \\
\hline Pessoas/ cômodo & 0,4388 & 0,0257 & $*$ & 1,551 \\
\hline Pessoas/ domic. 1 & 0,7417 & 0,0638 & $*$ & 2,100 \\
\hline 2 & 0,3003 & 0,0547 & $*$ & 1,350 \\
\hline 3 & $-0,0034$ & 0,0460 & $94,2 \%$ & 0,997 \\
\hline 5 ou 6 & 0,0559 & 0,0421 & $18,4 \%$ & 1,058 \\
\hline$\geq 7$ & 0,2925 & 0,0532 & $*$ & 1,340 \\
\hline Luz elétrica & 0,0342 & 0,0587 & $56,0 \%$ & 1,035 \\
\hline Água canalizada ${ }^{4}$ & $-0,4398$ & 0,0395 & $*$ & 0,644 \\
\hline Esgoto 5 & $-0,0522$ & 0,0332 & $11,6 \%$ & 0,949 \\
\hline Região ${ }^{6}$ Norte & 0,0012 & 0,0467 & $98,0 \%$ & 1,001 \\
\hline$M G+E S+R J$ & $-0,3592$ & 0,0419 & $*$ & 0,698 \\
\hline $\mathrm{SP}$ & $-0,2879$ & 0,0469 & $*$ & 0,750 \\
\hline Sul & $-0,1919$ & 0,0518 & $*$ & 0,825 \\
\hline Centro- 0 este & $-0,3270$ & 0,0606 & $*$ & 0,721 \\
\hline Área rural & $-0,6214$ & 0,0442 & $*$ & 0,537 \\
\hline Setor agrícola & $-0,1909$ & 0,0421 & $*$ & 0,826 \\
\hline PO (7) Mil. ou func.públ. & 0,1275 & 0,1110 & $25,1 \%$ & 1,136 \\
\hline Sem carteira ${ }^{8}$ & 0,6050 & 0,0526 & $*$ & 1,831 \\
\hline D oméstico & 0,4075 & 0,0732 & $*$ & 1,503 \\
\hline Conta própria & 0,4659 & 0,0500 & $*$ & 1,593 \\
\hline Empregador & $-0,1432$ & 0,1528 & $34,8 \%$ & 0,867 \\
\hline Inativos e outros & 0,3845 & 0,0548 & $*$ & 1,469 \\
\hline Apos. e pensões $>25 \% 9$ & $-0,1128$ & 0,0416 & $0,7 \%$ & 0,893 \\
\hline Tem pessoa $<18$ anos & $-0,6606$ & 0,0467 & $*$ & 0,517 \\
\hline
\end{tabular}

${ }^{1}$ Probabilidade caudal do teste da hipótese de nulidade do parâmetro. 0 asterisco assinala os casos em que essa probabilidade éinferior a $0,1 \%$.

${ }^{2}$ Renda domiciliar per capita : estratos delimitados em salários mínimos correntes, adotando como base 0 estrato com RD PC menor ou igual a 0,25 salário mínimo.

${ }^{3}$ Pessoa de referência do domicílio, adotando como base o homem branco com até 55 anos de idade.

${ }^{4}$ Tem água canalizada em pelo menos um cômodo.

${ }^{5}$ Escoadouro do banheiro ou sanitário ligado à rede coletora e/ ou fossa séptica.

${ }^{6}$ A região Nordeste é adotada como base.

${ }^{7}$ Posição na ocupação da pessoa de referência do domicílio, adotando como base os empregados com carteira.

${ }^{8}$ Empregados sem carteira ou sem declaração de carteira.

${ }^{9}$ A posentadorias e pensões constituem mais de $25 \%$ da renda domiciliar. 
Tabela 10. Modelo de lógite para a probabilidade de um domicílio ter insegurança alimentar leve, moderada ou grave. Brasil, 2004.

\begin{tabular}{|c|c|c|c|c|}
\hline Variável & $\begin{array}{l}\text { Estimativa do } \\
\text { parâmetro }\end{array}$ & $\begin{array}{l}\text { Estimativa do } \\
\text { desvio padrão }\end{array}$ & Prob. caudal ${ }^{1}$ & 0 dds ratio \\
\hline Constante & 1,2669 & 0,0717 & $*$ & - \\
\hline $\mathrm{RD} \mathrm{PC}^{2}(0,25 ; 0,5]$ & $-0,7165$ & 0,0322 & $*$ & 0,488 \\
\hline$(0,5 ; 1]$ & $-1,4905$ & 0,0320 & $*$ & 0,225 \\
\hline$(1 ; 2]$ & $-2,1497$ & 0,0352 & $*$ & 0,117 \\
\hline$(2 ; 3]$ & $-2,8331$ & 0,0471 & $*$ & 0,059 \\
\hline$(3 ; 5]$ & $-3,2851$ & 0,0588 & $*$ & 0,037 \\
\hline$(5 ; 10]$ & $-3,8599$ & 0,0907 & $*$ & 0,021 \\
\hline$>10$ & $-4,8249$ & 0,2090 & $*$ & 0,008 \\
\hline $\mathrm{PR}^{3}$ Escolaridade & $-0,0444$ & 0,0023 & $*$ & 0,957 \\
\hline $\mathrm{PR}^{3}$ Mulher & 0,4058 & 0,0194 & $*$ & 1,501 \\
\hline $\mathrm{PR}^{3}>55$ anos & $-0,1307$ & 0,0234 & $*$ & 0,877 \\
\hline PR3preto ou pardo & 0,3094 & 0,0164 & $*$ & 1,363 \\
\hline $\mathrm{PR}^{3}$ amarelo & $-0,1966$ & 0,1462 & $17,9 \%$ & 0,822 \\
\hline Pessoas/ cômodo & 0,5895 & 0,0268 & * & 1,803 \\
\hline Pessoas/ domic. 1 & 0,1341 & 0,0370 & $*$ & 1,144 \\
\hline 2 & 0,1312 & 0,0292 & $*$ & 1,140 \\
\hline 3 & 0,0855 & 0,0224 & $*$ & 1,089 \\
\hline 5 ou 6 & 0,0274 & 0,0235 & $24,5 \%$ & 1,028 \\
\hline$\geq 7$ & 0,0266 & 0,0394 & $50,0 \%$ & 1,027 \\
\hline Luz elétrica & $-0,1212$ & 0,0461 & $0,8 \%$ & 0,886 \\
\hline Água canalizada4 & $-0,3589$ & 0,0298 & $*$ & 0,698 \\
\hline Esgoto 5 & $-0,0763$ & 0,0197 & $*$ & 0,927 \\
\hline Região ${ }^{6}$ Norte & $-0,2251$ & 0,0310 & $*$ & 0,798 \\
\hline$M G+E S+R J$ & $-0,3068$ & 0,0227 & $*$ & 0,736 \\
\hline $\mathrm{SP}$ & $-0,4120$ & 0,0248 & * & 0,662 \\
\hline Sul & $-0,3796$ & 0,0267 & * & 0,684 \\
\hline Centro-O este & $-0,3724$ & 0,0317 & $*$ & 0,689 \\
\hline Área rural & $-0,6170$ & 0,0275 & $*$ & 0,540 \\
\hline Setor agrícola & $-0,1639$ & 0,0265 & $*$ & 0,849 \\
\hline $\mathrm{PO}^{7}$ Mil. ou func. Públ. & 0,0970 & 0,0418 & $2,0 \%$ & 1,102 \\
\hline Sem carteira ${ }^{8}$ & 0,4004 & 0,0265 & $*$ & 1,492 \\
\hline Doméstico & 0,1641 & 0,0427 & * & 1,178 \\
\hline Conta própria & 0,2683 & 0,0232 & $*$ & 1,308 \\
\hline Empregador & $-0,3261$ & 0,0536 & $*$ & 0,722 \\
\hline Inat. e outros & 0,1020 & 0,0274 & $*$ & 1,107 \\
\hline Apos. e pensões $>25 \% 9$ & 0,0392 & 0,0229 & $8,6 \%$ & 1,040 \\
\hline Tem pessoa $<18$ anos & $-0,0167$ & 0,0237 & $48,0 \%$ & 0,983 \\
\hline
\end{tabular}

${ }^{1}$ Probabilidade caudal do teste da hipótese de nulidade do parâmetro. 0 asterisco assinala os casos em que essa probabilidade é inferior a 0,1\%.

${ }^{2}$ Renda domiciliar per capita : estratos delimitados em salários mínimos correntes, adotando como base o estrato com RD PC menor ou igual a 0,25 salário mínimo.

${ }^{3}$ Pessoa de referência do domicílio, adotando como base o homem branco com até 55 anos de idade.

${ }^{4}$ Tem água canalizada em pelo menos um cômodo.

${ }^{5}$ Escoadouro do banheiro ou sanitário ligado à rede coletora e/ ou fossa séptica.

${ }^{6}$ A região Nordeste é adotada como base.

${ }^{7}$ Posição na ocupação da pessoa de referência do domicílio, adotando como base os empregados com carteira.

${ }^{8}$ Empregados sem carteira ou sem declaração de carteira.

${ }^{9}$ A posentadorias e pensões constituem mais de $25 \%$ da renda domiciliar. 
agregam as três categorias de insegurança alimentar (leve, moderada e grave), a odds ratio de SP, em comparação com o Nordeste, é 0,662.

Conforme os resultados apresentados na tabela 10, a existência de luz elétrica, água encanada e esgoto apropriado reduzem a probabilidade de insegurança alimentar. Q uando a análise se restringe à insegurança alimentar grave (tabela 9), apenas a água encanada, dentre estas três variáveis, tem efeito estatisticamente significativo. A influência dessas variáveis e as diferenças regionais mostram que a probabilidade de insegurança alimentar para um domicílio depende do acesso a serviços que são, em grande parte, dependentes da atuação do Estado (saneamento, fornecimento de água tratada, serviços de saúde, etc.). ${ }^{3}$

Foi visto, na tabela 4, que a probabilidade de segurança alimentar diminui sistematicamente com o aumento do número de pessoas por domicílio. Quando é controlado o efeito de outras variáveis, 0 quadro fica muito diferente, como mostram as tabelas 9 e 10. Note-se que foi adotado como base o valor de 4 pessoas por domicílio. Então, coeficientes positivos e significativos indicam maior probabilidade de insegurança alimentar, em comparação com essa base. Verifica-se que há maior probabilidade de insegurança alimentar para domicílios com apenas 1 ou 2 pessoas, e também há maior probabilidade de insegurança alimentar grave para domicílios com 7 ou mais pessoas.

Nas tabelas 2 e 6 foi visto que a residência rural (em comparação à urbana) e a atividade agrícola da pessoa de referência estão associadas a maior probabilidade de insegurança alimentar. Porém, isso está associado, em grande parte, às diferenças de RD PC e escolaridade. Os valores médios da RD PC nas áreas urbanas e rurais são, respectivamente, R\$ 533 e R\$232, e as escolaridades médias são 6,80 e 2,93. Quando se contrastam os domicílios cuja pessoa de referência é ocupada no setor agrícola com os demais, verifica-se que as médias da RD PC são R \$ 251 e R\$ 530 e as escolaridades médias são 2,77 e 6,84, respectivamente 4 . Assim, quando os efeitos de RD PC e escolaridade são controlados, juntamente com os efeitos de outras variáveis incluídas nos modelos das tabelas 9 e 10, verifica-se que residência rural e atividade agrícola contribuem para reduzir a insegurança alimentar. É necessário reconhecer que a dimensão desse efeito pode estar superestimada devido à maior subestimação das rendas agrícolas, particularmente 0 fato de 0 rendimento obtido na PNAD não incluir o valor da produção para autoconsumo. Por outro lado, é certo que, fixadas a RD PC e as demais variáveis incluídas no modelo de lógite, o acesso a alimentos é, em geral, mais fácil para pessoas com domicílio rural e ocupação agrícola.

Foram incluídas no modelo variáveis binárias para distinguir sete categorias de posição na ocupação da pessoa de referência do domicílio: empregado com carteira (adotado como base), militar ou funcionário público estatutário, outros empregados sem carteira ou sem declaração de carteira, trabalhador doméstico, conta própria, empregador e outras categorias (incluindo inativos). Verifica-se, na tabela 10, que os seis efeitos são estatisticamente significativos (pelo menos a 5\%), quando se considera a probabilidade de insegurança alimentar fraca, moderada ou grave. Na tabela 9, para insegurança alimentar grave, os coeficientes para "militar ou funcionário público estatutário" e "empregador" não são estatisticamente significativos. O s resultados são bastante coerentes e podem ser interpretados em função da estabilidade dos rendimentos em cada categoria. Empregados sem carteira e trabalhadores por conta própria, por exemplo, têm rendimentos mais instáveis do que empregados com carteira, 0 que leva a uma maior insegurança alimentar. Novamente, é necessário reconhecer que o controle pela RDPC é muito imperfeito e que isso pode estar causando uma superestimação dos efeitos específicos da "posição na ocupação". ${ }^{5}$

\footnotetext{
${ }^{3}$ Como já assinalado em estudos de prevalência de retardo de crescimento de crianças por Monteiro, Benício e G ouveia ${ }^{[3]}$ e Monteiro ${ }^{[4]}$. Ver também Hoffmann ${ }^{[5]}$.

4 Cabe ressaltar que a classificação rural-urbana não se confunde com a distinção entre agrícola e não-agrícola. Mais de 1/ 3 dos domicílios da área rural têm pessoa de referência que não está ocupada no setor agrícola.

${ }^{5} \mathrm{O}$ valor médio da RDPC é R $\$ 487$ para empregados com carteira, R\$ 873 para militares e funcionários públicos estatutários, $\mathrm{R} \$ 333$ para empregados sem carteira e sem declaração de carteira, $\mathrm{R} \$ 226$ para trabalhadores domésticos, R 397 para os conta própria, $\mathrm{R} \$ 1.301$ para empregadores e $\mathrm{R} \$ 444$ para a categoria residual.
} 
Uma das variáveis explanatórias incluídas capta o efeito de a pessoa de referência do domicílio ter mais de 55 anos de idade, uma característica de $28 \%$ dos domicílios. O coeficiente dessa variável é negativo e estatisticamente significativo, tanto na tabela 9 como na tabela 10. O utra variável incluída destaca os domicílios nos quais o rendimento de aposentadorias e pensões representa mais de 25\% do rendimento domiciliar, fato que ocorre em $29 \%$ dos domicílios. O coeficiente dessa variável não é estatisticamente significativo ao nível de $5 \%$, quando se considera 0 agregado das categorias de insegurança alimentar (leve, moderada ou grave), mas é negativo e significativo ao nível de $1 \%$, quando a variável dependente é a probabilidade de insegurança alimentar grave. Aqui há indicações de que, mesmo depois de controlado o efeito da RDPC, 0 rendimento de aposentadorias e pensões, pela sua estabilidade, contribui para reduzir a insegurança alimentar grave.

Já é conhecido o fato de que pretos e pardos, em comparação com brancos, têm RD PC média mais baixa ( $\mathrm{R} \$ 305$ contra $\mathrm{R} \$ 636)$ e escolaridade média mais baixa (4,98 contra 7,21 anos). Então é esperado que a prevalência de insegurança alimentar seja maior entre domicílios com pessoas de referência preta ou parda. A proporção de domicílios com insegurança alimentar grave é quase três vezes maior entre aqueles cuja pessoa de referência é preta ou parda (10,00\%), do que entre aqueles cuja pessoa de referência é branca (3,55\%). Mas, mesmo quando se controla o efeito da RD PC, da escolaridade e de outras variáveis, permanece um efeito estatisticamente significativo de cor preta ou parda determinando maior insegurança alimentar (aumento de $41,8 \%$ no odds de insegurança alimentar grave), sugerindo a existência de efeitos específicos associados à discriminação. Aqui também é necessário lembrar que o coeficiente para cor preta ou parda pode estar superestimado, devido às limitações na mensuração das variáveis de controle, particularmente a RD PC.

$\mathrm{Na}$ tabela 3, verificou-se que a presença de pessoas com menos de 18 anos de idade aumenta, substancialmente, a probabilidade de insegurança alimentar. ${ }^{6}$ Mas isso está fortemente associado à RD PC, cujo valor médio é R\$316, quando o domicílio tem pelo menos uma pessoa com menos de 18 anos, e R\$ 741 em situações opostas. Nos modelos de lógite das tabelas 9 e 10, nos quais se controla o efeito da RDPC e de outras variáveis, verifica-se que a presença de pessoa com menos de 18 anos reduz a probabilidade de insegurança alimentar grave e não tem efeito estatisticamente significativo no agregado das três categorias de insegurança alimentar (leve, moderada ou grave). Cabe investigar melhor a razão pela qual, depois de controlados os efeitos das demais variáveis, a presença de pessoas com menos de 18 anos contribui para reduzir a insegurança alimentar grave. Uma explicação seria a existência de economias de escala no consumo (que poderiam ser consideradas, utilizando a renda domiciliar por adulto-equivalente em lugar da RD PC).

\section{Variações na estimativa da probabilidade de insegurança alimentar grave}

As equações de lógite ajustadas permitem estimar a probabilidade de um domicílio apresentar insegurança alimentar para qualquer situação hipotética definida por meio das variáveis explanatórias. Vamos fazer esse exercício utilizando os coeficientes da equação apresentada na tabela 9, referente à insegurança alimentar grave. Nesta seção, a probabilidade estimada de um domicílio ter insegurança alimentar grave é indicada por $\mathrm{P}$.

Como base considera-se um domicílio com $\mathrm{RDPC}$ de 0 a 1/ 4 de salário mínimo, com pessoa de menos de 18 anos de idade, participação de aposentadorias e pensões na renda domiciliar igual ou inferior a 25\%, 0,6 pessoas por cômodo, 4 pessoas por domicílio, com luz elétrica, água encanada e esgoto apropriado, na área urbana do Nordeste, cuja pessoa de referência é um homem branco, com escolaridade igual a 5, idade igual ou inferior a 55 anos, empregado com carteira em setor não-agrícola. Neste caso $P=9,72 \%$.

Se, a partir dessa base, for alterada apenas a região de residência, obtém-se $\mathrm{P}=9,73 \%$ no Norte,

\footnotetext{
${ }^{6}$ Ver também IBGE E ${ }^{[1]}$, p. 38.
} 
$\mathrm{P}=6,99 \%$ no conjunto $\mathrm{MG}+\mathrm{ES}+\mathrm{RJ}, \quad \mathrm{P}=7,47 \%$ em SP, $P=8,16 \%$ no Sul e $P=7,21 \%$ no CentroO este.

Partindo da mesma base e alterando apenas 0 estrato de RDPC, obtém-se $\mathrm{P}=4,80 \%$ para $0,25<\mathrm{RDPC}<0,5$ salário mínimo, $\mathrm{P}=1,00 \%$ para $1<\mathrm{RDPC}<2$ salários mínimos e P = 0,02\% para RDPC > 10 salários mínimos. Note-se que a probabilidade de insegurança alimentar grave se reduz a menos da metade quando se passa do primeiro para o segundo estrato de RD PC e se torna desprezível para o estrato mais rico.

O valor de $\mathrm{P}$ aumenta de $9,72 \%$ para $13,78 \%$, se a pessoa de referência for mulher.

O valor de P aumenta de 9,72\% para 13,25\%, se a pessoa de referência for preta ou parda.

A ausência de água encanada faz 0 valor de $\mathrm{P}$ aumentar de 9,72\% para $14,32 \%$.

Partindo da situação adotada como base, se 0 número de pessoas por cômodo abaixar de 0,6 para 0,25 , P diminui de $9,72 \%$ para $8,45 \%$, e se 0 número de pessoas por cômodo aumentar para 1,5, o valor de P sobe para $13,78 \%$.

É interessante considerar duas situações extremas, para mostrar a importância do conjunto de determinantes da insegurança alimentar analisado neste trabalho. Em uma situação desfavorável, com pessoa de referência sem nenhuma escolaridade, mulher, cor preta ou parda, empregada sem carteira, domicílio com 7 pessoas ou mais, 2 pessoas por cômodo, sem luz elétrica, sem água encanada e sem esgoto apropriado, sem pessoa com menos de 18 anos de idade e demais características iguais às da situação básica, temos $\mathrm{P}=82,67 \%$, ou seja, a probabilidade de ocorrer insegurança alimentar grave supera 4/5. No outro extremo, para um domicílio no estrato de RDPC mais elevada, cuja pessoa de referência tem escolaridade igual ou superior a 15 anos, mais de 55 anos de idade e é um empregador no setor agrícola (tipicamente um fazendeiro) na área rural de SP, com 0,25 pessoas por cômodo e demais características iguais às da situação básica, teremos 0 valor de P praticamente igual a zero.

\section{Considerações finais}

A medida de insegurança alimentar utilizada neste trabalho é baseada na Escala Brasileira de Insegurança Alimentar, elaborada pelo IBGE, com base em 15 perguntas respondidas por pessoa do domicílio. Cabe ressaltar que há um evidente elemento subjetivo nessa medida, sendo que a primeira pergunta é se "Moradores tiveram preocupação de que os alimentos acabassem antes de poderem comprar ou receber mais comida".

O s determinantes da insegurança alimentar analisados neste trabalho permitem explicar, em geral, as grandes variações observadas na probabilidade de insegurança alimentar. 0 determinante isolado mais importante é, sem dúvida, a renda domiciliar per capita (RDPC). É muito substancial a redução na probabilidade de insegurança alimentar grave associada à passagem do estrato mais pobre (com $\mathrm{RDPC} \leq 0,25$ salário mínimo) para 0 estrato seguinte (com RD PC de mais de 0,25 a 0,5 salário mínimo), e essa probabilidade se torna desprezível no estrato de RD PC > 10 salários mínimos.

Vários trabalhos mostraram que os programas de transferência de renda, como o Bolsa Família, são muito bem focalizados e tiveram um papel importante na redução da pobreza no Brasil no período recente (particularmente a partir de 2001) ${ }^{7}$. Conclui-se, portanto, que tais programas têm efeito importante na segurança alimentar das famílias pobres.

Mesmo controlando o efeito da RD PC, verifica-se que há diferenças regionais e efeitos importantes da escolaridade da pessoa de referência do domicílio e da existência de água encanada, 0 que mostra a relevância da atuação do Estado, promovendo serviços básicos de fornecimento de água, saneamento, educação, etc.

Um aspecto importante deste trabalho é mostrar vários casos em que o sentido do efeito de um fator é alterado quando se controla o efeito de

${ }^{7}$ Ver Soares et al. ${ }^{[6]}$, Hoffmann ${ }^{[7]}$, Soares et al. ${ }^{[8]}$ e Medeiros et al. ${ }^{[9]}$. 
outros fatores, particularmente da RDPC. Se, por exemplo, simplesmente forem comparadas áreas urbanas e rurais, a insegurança alimentar é maior nas últimas. Mas, quando é controlado o efeito de outras variáveis, particularmente da RDPC e da escolaridade da pessoa de referência do domicílio, verifica-se que a insegurança alimentar tende a ser menor nas áreas rurais. Fenômeno semelhante ocorre na análise do efeito de o setor de atividade da pessoa de referência ser ou não agrícola.

Quando simplesmente são comparados domicílios com pelo menos uma pessoa menor de 18 anos de idade com domicílios sem pessoas nesta faixa de idade, observa-se que a insegurança alimentar é maior nos primeiros. Entretanto, no modelo de lógite, com controle para o efeito de outras variáveis, a presença de pessoas com menos de 18 anos de idade no domicílio contribui para reduzir a probabilidade de insegurança alimentar grave, indicando que o aumento da insegurança alimentar, observado na análise anterior, ocorre essencialmente por causa da redução da RD PC, pois a presença de crianças e jovens eleva o número de pessoas por domicílio e contribui pouco para aumentar a renda domiciliar.

\section{Referências bibliográficas}

[1] IBG E. Segurança alimentar: 2004. Rio de Janeiro, Instituto Brasileiro de G eografia e Estatística. 2006.

[2] Sen, A. Poverty and famines: an essay on entitlement and deprivation. Oxford University Press. 1981.

[3] Monteiro, C.A.; Benício, M.H. e Gouveia, N.C. Saúde e nutrição das crianças brasileiras no final da década de 80. In: perfil estatístico de crianças e mães no Brasil: aspectos de saúde e nutrição de crianças no Brasil, 1989. IBGE, 1992.
[4] Monteiro, C.A. O mapa da pobreza no Brasil. Cadernos de N utrição 4, p. 1-6.1992.

[5] Hoffmann, R. Pobreza, insegurança alimentar e desnutrição no Brasil. E studos A vançados 9 (24)159172, maio- agosto de 1995.

[6] Soares, F.V.; Soares,S.; Medeiros,M. e O sório,R.G. Cash transfer programmes in Brazil: impacts on inequality and poverty International Poverty Centre,UNDP/ IPEA, Working Paper n.21, junho de 2006.

[7] Hoffmann, R. Transferências de renda e a redução da desigualdade no Brasil e cinco regiões entre 1997 e 2004. E conômica, Rio de Janeiro, v.8, n.1, p.55-81, junho de 2006.

[8] Soares,S.; O sório,R.G.;Soares,F.V.; Medeiros, M. eZepeda,E. Conditional cash transfers in Brazil, Chile and México: impacts upon inequality. International Poverty Centre, UND P/ O PES, Working Paper n.35, abril de 2007.

[9] Medeiros, M.; Brito,T.e Soares, F. Programas focalizados de transferência de renda no Brasil: contribuições para o debate. IPEA, Brasília,Texto para Discussão n. 1283, junho de 2007.

\section{Autor}

Rodolfo Hoffmann - Professor do Instituto de Economia da UNICAMP, com apoio do CNPq.

Correspondência: Endereço do contato: Instituto de Economia- UNICAMP - Caixa postal 6135, CEP: 13083857 - Campinas, SP. E-mail: rhoffman@ eco.unicamp.br

Recebido em: 06/ 09/ 2007

Aceito em: 03/ 04/ 2008 